Accelerator Development Department

$\mathrm{AD} / \mathrm{RHIC}-46$

BROOKHAVEN NATIONAL LABORATORY

Associated Universities, Inc.

Upton, New York 11973

RHIC TECHNical Note No. 46

RADIATION FROM MUONS AT RHIC

A. Stevens

$2 / 1 / 89$ 


\title{
RADIATION FROM MUONS AT RHIC
}

\author{
A. Stevens \\ Brookhaven National Laboratory \\ Upton, New York 11973
}

\section{Introduction}

Possible effects on both personnel and the environment from radiation resulting from beam loss at RHIC have been previously evaluated ${ }^{(1,2,3)}$ and found to be well below DOE guideliness. The only radiation source which was not considered in these evaluations are muons which can, in principle, penetrate considerable distances in accelerator shielding materials. In the proposed ISABELLE accelerator for example, calculations showed that a $0.1 \%$ beam loss in certain locations would result in radiation levels at the site boundary which approached or exceeded the $5 \mathrm{mRem} /$ year limit. ${ }^{(4)}$

However, the muon shielding surrounding the RHIC tunnel was designed for ISABELLE which would have had an annual accelerated energy greater than 10 times that of RHIC and a significantly greater maximum energy per nucleon (400 GeV contrasted with RHIC protons at $250 \mathrm{GeV}$ ). A priori we therefore anticipate a negligible problem from muons in RHIC. The calculations presented here validate this expectation.

\section{Method And Geometry of Calculation}

Muon dose was calculated using a version of the hadronic cascade Monte Carlo program CASIM $^{(5,6)}$ modified to calculate energy deposition from muons resulting from three sources: (1) pion decay, (2) kaon decay, and (3) direct production. The energy loss mechanism of muons in matter is discussed elsewhere. ${ }^{(7)}$ Measurements of muon flux exterior to the earth shielding at FNAL have been made and found to agree with CASIM predictions to better than a factor of $2 .^{(8)}$

The earth shielding around the RHIC tunnel, including muon "lobes" which reach their maximum radial extension at the center of each arc, is shown in Fig. 1. CASIM calculates energy deposition from muons in finite bins. Figure 2 shows the approximation made of the RHIC tunnel and the radial bins used in the calculation made here. The bin sizes are $2.5 \mathrm{~m}$ (radial) by $0.5 \mathrm{~m}$ (vertical) by $-8.5 \mathrm{~m}$ (beam direction). The number of bins in the radial direction varies from 10 at the entrance to the "regular lattice" to 35 at the midpoint of the arc. As shown in Fig. 2, the tunnel radius is taken as $2.5 \mathrm{~m}$, the "expanded" tunnel section being ignored. Further approximations adopted in describing the magnet lattice are described in Appendix A.

Three locations have been considered as sources of beam interactions: (a) the crossing point, (b) a point on the vacuum pipe 20.5 meters upstream of the entrance to 
the regular lattice, and (c) the beam dump which is located approximately 75 meters upstream of the crossing point. The crossing point geometry is intended to simulate beam-beam interactions. Although the version of CASIM used for the calculations presented here does not explicitly simulate beam-beam interactions, a beam-stationary target model gives similar results in the forward direction and it is from forward pions that the most penetrating muons arise. The second location is intended to simulate loss on a limiting aperture collimator (LAC). Although the number and locations of such devices has not yet been defined, the location considered here has 3 advantageous features: (1) it is downstream of the crossing point, (2) it is a location of high dispersion $(-1.1 \mathrm{~cm}$ per percent momentum difference) and therefore a good location for intercepting off-momentum ions and (3) the -21 meter drift space before the start of the regular lattice minimizes the probability of quenching magnets from secondaries emerging from the collimator. The physical material of the collimator has been ignored;interactions are forced to occur in the vacuum chamber. Results from this location therefore represent upper limits. The last location, the internal dump, has been described elsewhere. ${ }^{(9)}$

Having defined the assumed source locations, it remains to defined the source strength, i.e., the beam "lost" per year at each of the three points considered.

\section{Assumed Beam Loss}

For studies of potential radiation problems from hadrons, a conservative scenario was adopted which assumes $8.6 \times 10^{14} \mathrm{Au}$ ions per year are accelerated to $100 \mathrm{GeV} /$ nucleon in RHIC. The scenario is described in detail in Appendix A of Reference 2. This scenario would give zero muon flux outside the shielding; calculations assuming an incident beam of $100 \mathrm{GeV} / \mathrm{A}$ ions resulted in muon trajectories which reached the end of their range in the earth lobe. However, non-zero results are obtained for muons exiting the lobe if incident $250 \mathrm{GeV} / \mathrm{c}$ protons are considered. Assumptions are therefore required concerning the number of protons per year accelerated in RHIC.

For both convenience and consistency, we generally follow the scenario outlined in Ref. 2. We therefore retain the assumption of 1710 fills per ring per year, but now assume that one-third of the fills contain protons for $p$,Nucleus collisions. This gives 346 proton fills for operations and 224 fills for studies per year. Of the operations fills, half are for set-up and are assumed of $1 \mathrm{hr}$. duration and half are for physics and are assumed to last 10 hours. All the studies fills are assumed to last 1 hour. We retain the conservative assumption that each fill contains 4 times the original design intensity, in this case $2.28 \times 10^{13}$ protons. Retaining further the aperture and RF loss assumptions given in Ref. 2 (3\% for the physics runs, $0.45 \%$ for set-up, $0.6 \%$ for studies) and assuming a proton, $\mathrm{Au}$ crossing point loss of $6.9 \%$ in 1 hour and $30 \%$ in 10 hours (the 
nonlinearity being due to emittance blow-up of the heavy ion beam) we derive the following values for proton loss per year:

\begin{tabular}{lrl}
\hline LAC & $8.3 \times 10^{13}$ & $(1 / 2$ total $)$ \\
Crossing Point & $3.0 \times 10^{14}$ & $(1 / 6$ total $)$ \\
Dump & $11.0 \times 10^{15}$ & \\
\hline
\end{tabular}

The Limiting Aperture Collimator value is one half of the total lost on LACs because several will presumably be present. The loss values for the beam-beam interaction rate given above assumes 6 crossing points. Since loss at each crossing point "points at" a different position on the site boundary only loss at a specific crossing point is of interest. Approximately 3 times more protons are lost at a crossing point than at a limiting aperture collimator. This contrasts with the estimate in Ref. 2 that approximately 20 times more $\mathrm{Au}$ ions (from $\mathrm{Au}, \mathrm{Au}$ collisions) would be lost on LACs than at the crossing points. The reason for this difference is that $\mathrm{Au}, \mathrm{Au}$ collisions at the crossing point are dominated by Coulomb processes, the products of which are assumed to interact on the LACs. In point of fact, neither the conclusions reached in this note nor the radiological estimates made in Ref. 2 would be altered if the amount of beam undergoing nuclear interactions at the crossing point and at an LAC were reversed.

\section{Results}

CASIM propagates the hadronic cascade through the magnetic lattice, creating a muon "weight" at each interaction for each of the three muon sources (direct, pion decay, kaon decay) mentioned above. One expects that pions created in the first generation (from $250 \mathrm{GeV} / \mathrm{c}$ protons) decaying into relatively high energy muons will dominate the muon dose at deep penetration distances in the muon lobe. In the ISABELLE muon dose estimates, ${ }^{(4)}$ this was, in fact, the only source considered. To compare this component with muons coming from all sources, a version of CASIM was created which discarded all muons other than those resulting form first generation pion decay. The comparison is shown in Fig. 3 which plots dose as a function of radial shielding at the center of the arc for both versions of the program for the crossing point source geometry. The points at small radial distances which appear only in the complete-cascade version of the program are an artifact of the regular lattice approximation described in Appendix A; because the focussing properties of the regular lattice have been ignored, fast forward protons, inaccurately transported, interact in magnets well within the regular lattice and give rise to these muons. At higher radial depths, the two versions of CASIM agree very well, justifying the usual assumption of first generation pion decay dominance. The two peaks in Fig. 3, at radial distances of -47 meters and 69 meters are better regarded as one peak with a "hole" created by absorption of the magnets in the insertion region; similar distributions have been previously noted. ${ }^{(10)}$ 
In most of the runs made, simulation of the earth lobe was made only to the center of the arc. Results at the exit point of the lobe were obtained by extrapolation. Some later runs were made which verified the extrapolation procedure.

As described in Ref. 9, energy emerging from the dump is a sensitive function of displacement of the beam on the dump face. The dump geometry also presents the longest lattice and therefore the greatest problem as concerns computer time. Runs were made at only $1 \mathrm{~mm}$ and $1 \mathrm{~cm}$ displacements.

The maximum emerging dose in Rem per interacting proton for the three source points considered was found to be the following:

\begin{tabular}{ll}
\hline LAC & $1.7 \times 10^{-18}$ \\
Crossing Point & $3.5 \times 10^{-18}$ \\
Dump: $(1 \mathrm{~mm})$ & $1.4 \times 10^{-18}$ \\
$\quad(1 \mathrm{~cm})$ & $1.4 \times 10^{-19}$ \\
\hline
\end{tabular}

The statistical error is estimated to be about $50 \%$.

With the loss as estimated in the previous section, the LAC and crossing point sources combine to give $1.1 \mathrm{mRem}$ per year emerging from the berm. As discussed in Ref. 9 and elsewhere, ${ }^{(11)}$ the RHIC dump system is designed to achieve a profile whose displacement on the dump face extends from 1.0 to $1.5 \mathrm{~cm}$. A reasonable estimate of the average emerging dose per proton with this profile would then be $1.1 \times 10^{-19} \mathrm{Rem}$. Combining this with our estimate of beam "loss" on the dump gives $1.2 \mathrm{mRem}$ per year emerging from the shielding beam.

The dose at the site boundary is attenuated by $1 / \mathrm{r}^{2}$ from the emerging dose. Conservatively taking $\mathrm{r}=0$ at the point of point of proton interaction gives solid angle reduction factors which vary according to both the source location and the intersection region around the RHIC site. From Ref. 4, the nearest point (along the beam direction) to the site boundary occurs at 10 o'clock in the clockwise direction and is 650 meters measured from the crossing point. The solid angle reduction factors here are 0.26 for crossing point loss and 0.16 for LAC loss. These sources add to give -0.29 mRem per year at this point on the site boundary. The other special location, because of the location of the internal dump, is at $6 \mathrm{o}^{\prime}$ clock in the clockwise direction. The site boundary here is 1030 meters from the crossing point and solid angle factors vary from 0.14 for loss on the dump to 0.054 for loss on an LAC. All three sources are additive here with essentially the same results, $0.28 \mathrm{mRem}$ per year.

\section{Summary/Conclusions}

We estimate muon dose at the site boundary from beam loss at RHIC to be about 0.3 mRem per year, well under the $5 \mathrm{mRem}$ per year criteria. The muon dose comes entirely 
from proton running; muons from $100 \mathrm{GeV} /$ nucleon heavy ions do not penetrate the earth shielding. This estimate contains a number of conservative assumptions, including deliberately high estimates of both proton beam intensity and the fraction of time devoted to proton running as well as neglect of significant amounts of shielding which will in fact exist within the RHIC tunnel. This estimate should therefore be well above what is actually expected to occur.

\section{Acknowledgements}

The author thanks Andy Van Ginneken (FNAL) for providing the version of CASIM code used in the calculations presented here and for several enlightening conversations related to the details of this code. Gratitude is also extended to Alan Thorndike for assistance in reconstructing what had been done as regards muon radiation calculations for ISABELLE.

\section{References}

1. "Environmental Analysis Report Relativistic Heavy Ion Collider", Dames \& Moore, July, 1988.

2. A.J. Stevens, "Radioisotope Production in Air and Soil in RHIC", RHIC Technical Note \#29, Nov., 1987.

3. "Conceptual Design Report 4A; Relativistic Heavy Ion Collider", 1989 (in preparation).

4. A.J. Stevens and A.M. Thorndike, "Muon Shielding Requirements for Present Configuration", ISABELLE Project Tech. Note No. 65, 1978.

5. A. Van Ginneken, "CASIM Program to Simulate Hadronic Cascades in Bulk Matter", Fermilab FN-272, 1975.

6. A.J. Stevens, "Improvements in CASIM; Comparison with Data", AGS/AD/Tech. Note No. 296, 1988.

7. A. Van Ginneken, "Energy Loss and Angular Characteristics of High Energy Electromagnetic Processes", Nucl. Inst. and Meth. A251, 1986, pp. 21-39.

8. A.J. Elwyn, J.D. Cossairt, and W.S. Freeman, "The Monitoring of AcceleratorProduced Muons at Fermilab", Proceedings of the 22nd Topical Meeting on Instrumentation, Health Physics Society, San Antonio, Texas, December, 1988.

9. A.J. Stevens, "RHIC Internal Beam Dump; Preliminary Conceptual Design", RHIC Technical Note \# 43, Sept., 1988.

10. A.J. Stevens and A.M. Thorndike, "Comments on Muon Trapping", BNL 50740, June, 1977.

11. J. Claus and H. Foelsche, "Internal Beam Dump System for RHIC", RHIC Technical Note \# 45, Oct., 1988.

12. J. Claus, "Private Communication". 

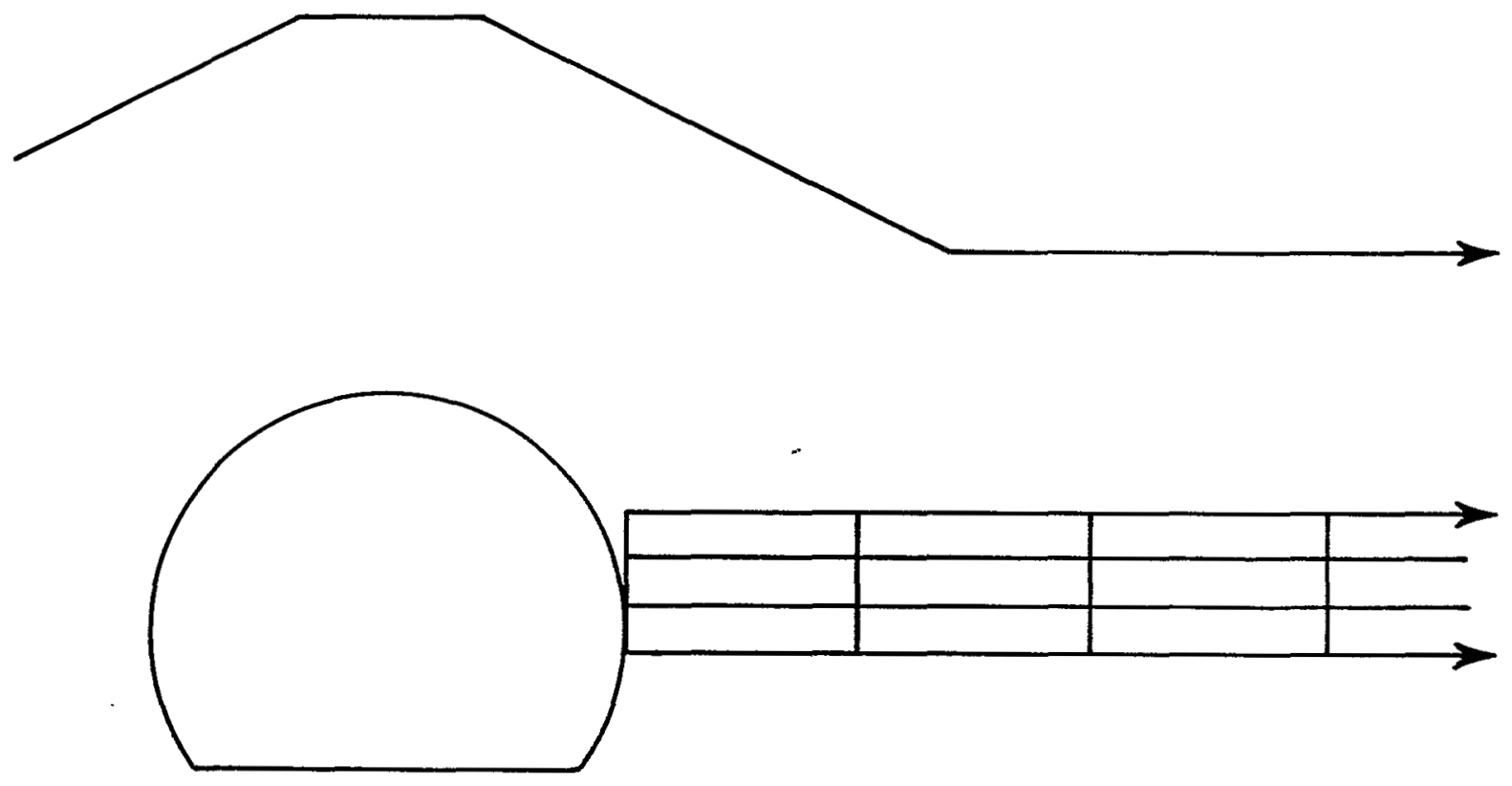

SCALE $\longrightarrow \quad K \longleftarrow=1$ METER

Fig. 2. Cutaway view of muon lobe showing Radial bins used in CASIM.

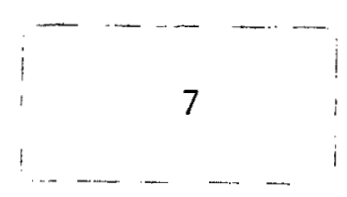




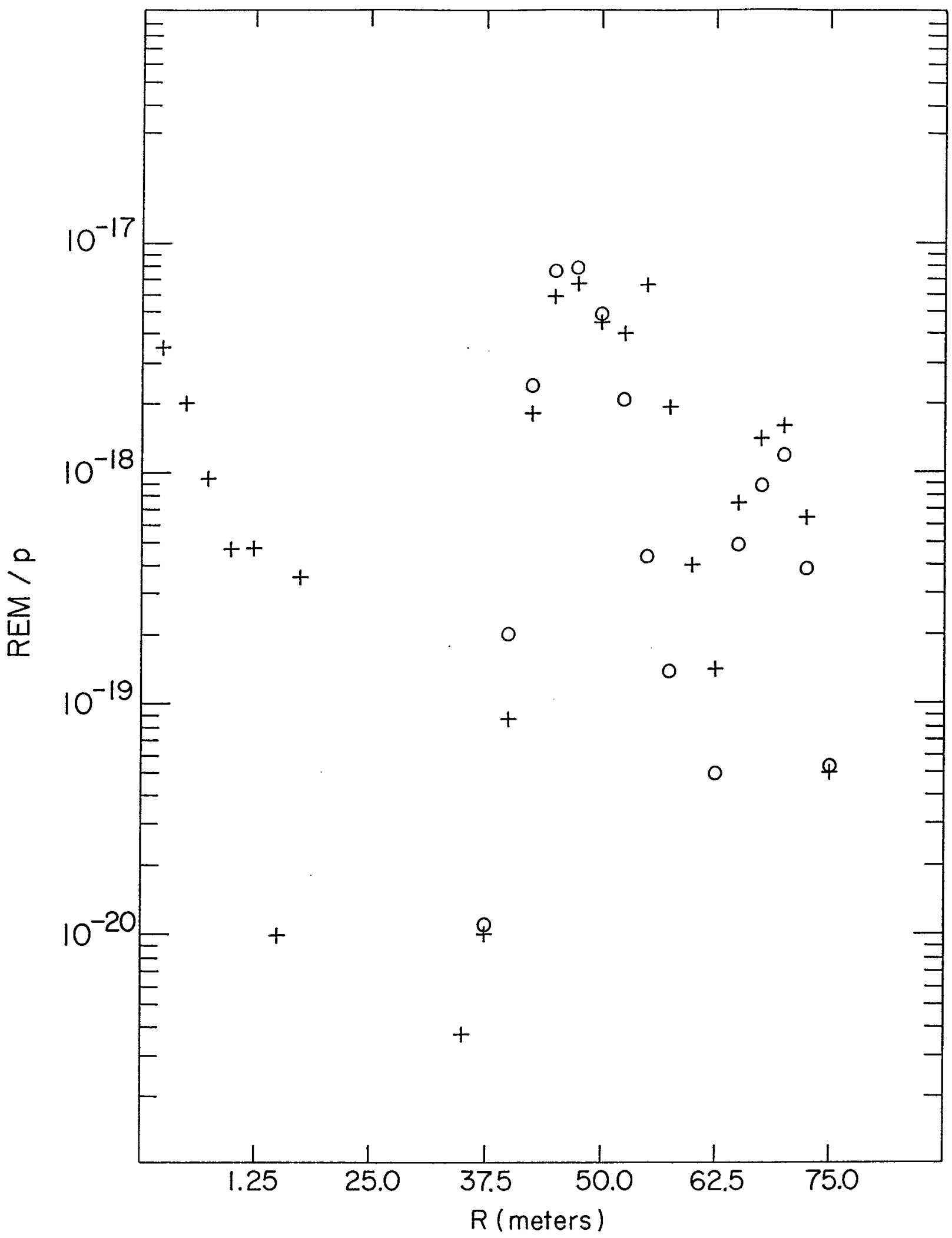

Fig. 3. Muon dose versus radial shielding depth at arc center. Circles represent dose from first generation pion decay. Crosses represent dose from full cascade. The vertical bins shown in Fig. 2 have been averaged. The loss source here is proton, iron collisions at the crossing point. 


\section{APPENDIX A \\ APPROXIMATION OF RHIC MAGNETS}

Only the ring in which beam is lost is simulated; the presence of a second nearby ring being always ignored.

The physical structure in the transverse plane is approximated by vacuum to a radius of $3.8 \mathrm{~cm}$, iron to $13 \mathrm{~cm}$, and iron with $2 \%$ of normal density to $30 \mathrm{~cm}$. The last radial region is intended to crudely simulate the presence of the cryostat.

In the insertion regions the lattice parameters, i.e., the positions, strengths, and lengths of dipoles and quadrupoles are assumed to be those corresponding to a symmetric $\beta$ of $6 \mathrm{~m}$. at the crossing point. ${ }^{(12)}$ The "ideal fields" are assumed everywhere within the $3.8 \mathrm{~cm}$ aperture. In the coil/yoke region $(3.8<\mathrm{r}<13 \mathrm{~cm}$.) we approximate the field by a crude model illustrated by the sketches shown in Fig. A1. The primary thrust of the model is to obtain approximately correct field directions in the yoke. From the returning flux lines shown in Fig. A1, we have:

$$
\begin{aligned}
& \hat{\mathrm{B}}(\text { dipole })=\mathrm{F}(\mathrm{r})^{*}[-\sin (\Theta) \hat{\mathrm{e}}(\mathrm{r})+\cos (\Theta) \hat{\mathrm{e}}(\Theta)] \\
& \hat{\mathrm{B}}(\text { quad })=\mathrm{G}(\mathrm{r})^{*}[-\sin (2 \Theta) \hat{\mathrm{e}}(\mathrm{r})+\cos (2 \Theta) \hat{\mathrm{e}}(\Theta)]
\end{aligned}
$$

where $\hat{\mathrm{e}}(\mathrm{r})$ and $\hat{\mathrm{e}}(\Theta)$ are unit vectors in the normal polar coordinate system.

For the field magnitudes the following expressions are used

$$
\begin{gathered}
\mathrm{F}(\mathrm{r})=\text { const }=-\mathrm{BO} * \mathrm{R} 1 /(\mathrm{R} 2-\mathrm{R} 1) \\
\mathrm{G}(\mathrm{r})=-\mathrm{K} * \mathrm{R} 1 * * 2 *(\mathrm{r}-\mathrm{R} 1) /(\mathrm{R} 2-\mathrm{R} 1) * * 2
\end{gathered}
$$

where $\mathrm{R} 1=3.8 \mathrm{~cm}$., $\mathrm{R} 2=13 \mathrm{~cm}, \mathrm{BO}=$ dipole central field, and $\mathrm{K}=$ quadrupole gradient. Both $\mathrm{BO}$ and $\mathrm{K}$ are signed quantities, $\mathrm{BO}$ being negative in the coordinate system used and $\mathrm{K}$ being negative for the horizontally focussing quadrupole shown in Fig A1. These expressions conserve the magnetic flux on the magnet midplane. An abrupt discontinuity in the field occurs at $\mathrm{R} 1$. In reality, the field on the midplane reverses in the region of the coil and the discontinuity arises as a consequence of not explicitly considering the coil to be a separate region. The integrated field seen by a muon traversing a magnet yoke should, however, be approximately correct.

The regular lattice, which begins at the magnet designated $\mathrm{Q}$, is approximated by one long dipole. 

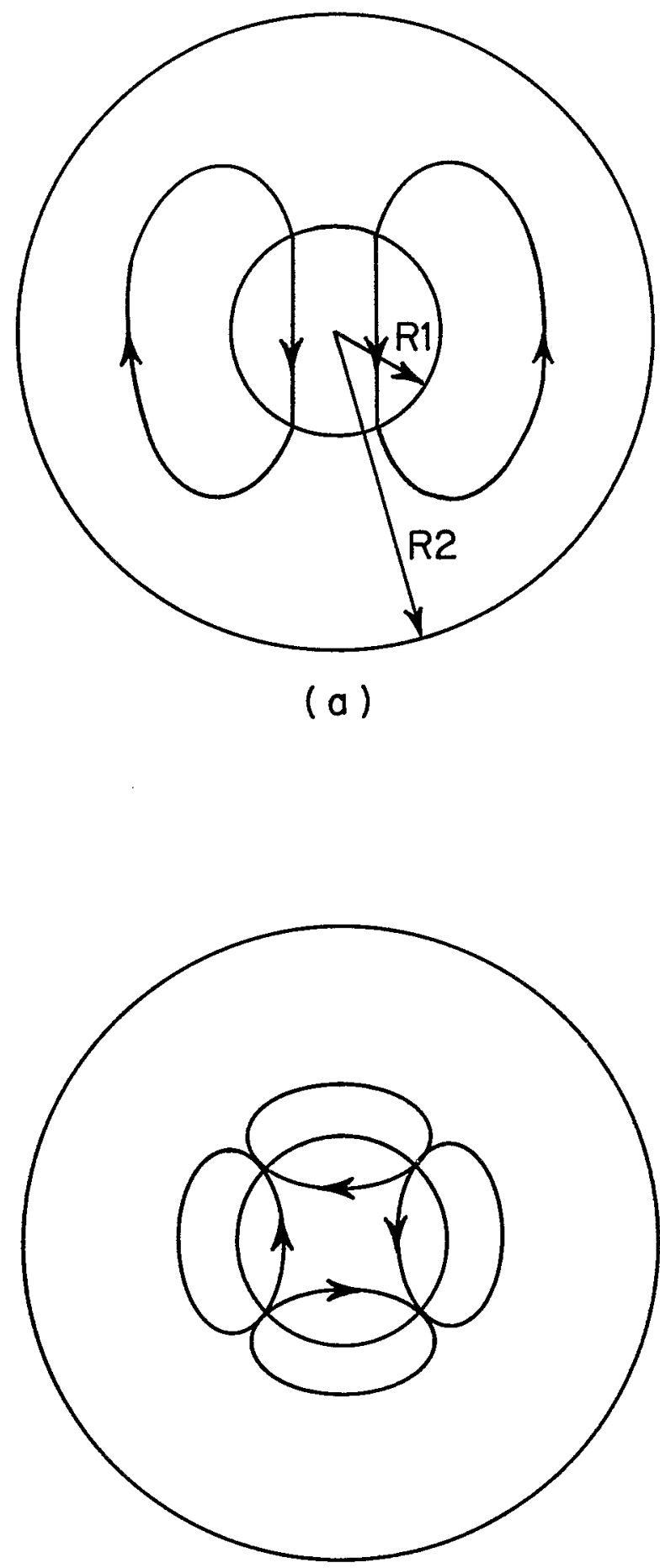

(b)

Fig. A1. Sketch of 2-dimensional magnet approximation showing "typical" flux lines for (a) dipole and (b) quadrupole

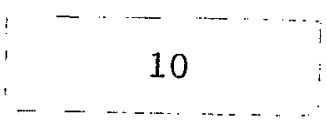

